\title{
REVISTA BIO-GRAFÍA. Escritos sobre la Biología y su enseñanza.
}

ISSN 2027-1034

\section{MISIÓN}

socializar las producciones de docentes en formación inicial y profesores-investigadores, sobre las innovaciones, experiencias, reflexiones, propuestas e investigaciones referentes a la Enseñanza de la Biología.

\section{OBJETIVOS}

Contribuir al fortalecimiento de la comunidad académica del campo de la Enseñanza de la Biología, especialmente en el ámbito colombiano y latinoamericano, mediante la socialización de innovaciones, experiencias, reflexiones, propuestas e investigaciones.

Constituir una estrategia de integración entre los diferentes actores implicados en las acciones, reflexiones y teorizaciones sobre la Biología y su Enseñanza.

Contribuir a la divulgación de eventos relacionados con la Enseñanza de la Biología y de las Ciencias Naturales.

Aportar a la formación inicial y permanente de profesores de Biología.

\section{TEMÁTICA}

En este medio se publican resultados de investigaciones, revisiones teóricas, innovaciones, reflexiones y experiencias sobre Biología y Educación en Biología. Para el caso de las investigaciones biológicas, cabe aclarar que es necesario incluir las implicaciones educativas de las mismas.

Así mismo, en la Revista se publican resúmenes de trabajos de grado y tesis (niveles de pregrado y postgrado).

\section{PERIODICIDAD: Semestral}

\section{ACCESO A LA REVISTA EN INTERNET}

Ingresando por cualquiera de las siguientes opciones:

- $\quad$ Revista Bio-grafia: Escritos sobre la Biologia y su Enseñanza.

- http://www.pedagogica.edu.co/revistas/ojs/index.php/bio-grafia.

- Mediante la siguiente ruta: Página Universidad Pedagógica Nacional, Fondo editorial, Revistas Electrónicas, Bio-grafía.

\section{REQUISITOS PARA LA PRESENTACIÓN DE LOS TEXTOS}

Los textos se deben presentar: en letra arial, tamaño 11, a espacio sencillo, a una columna, y con la extensión que se especifica en las diferentes secciones. Las figuras y tablas deben ir dentro del escrito.

Las citas bibliográficas dentro del texto, así como las referencias en la bibliografía, han de seguir las normas APA (ver apartado Presentación de la Bibliografía). Todas las citas bibliográficas deben corresponderse con las referencias bibliográficas presentadas al final del texto en orden alfabético de apellidos, en el apartado correspondiente a Bibliografía. 


\section{A DÓNDE REMITIR LOS ESCRITOS.}

Calle 72 \# 11-86, Bogotá (Colombia). Universidad Pedagógica Nacional, Departamento de Biología, oficina B319. Correos electrónicos: biografiadbi@gmail.com, biografia@ pedagogica.edu.co.

Para autores ubicados en la ciudad de Bogotá, se solicita además de enviar el escrito por correo electrónico, hacer entrega de una copia impresa (en sobre de manila sellado, dirigido al Comité Editorial de la Revista, incluyendo el correo electrónico). Para el caso de los artículos remitidos desde fuera de Bogotá, basta con la versión electrónica.

Es importante que los autores especifiquen la sección a la cual postulan el escrito, así como su afiliación (lugar de trabajo o estudio).

\section{SECCIONES EN LAS QUE SE PUEDE POSTULAR EL ESCRITO ${ }^{1}$}

A continuación se describen brevemente las características de las secciones de la revista.

- Bioartículo-investigaciones Presenta, de manera detallada, los resultados originales de investigaciones.

- Bioartículo-reflexiones Presenta reflexiones sobre experiencias o innovaciones educativas, apoyadas sólidamente en referentes teóricos.

- Bioartículo-revisiones. Presenta resultados de sistematizaciones obtenidas a partir de la revisión bibliográfica de un tema con el fin de dar cuenta de los avances y las tendencias de su desarrollo. Se caracteriza por presentar una cuidadosa revisión de por lo menos 50 referencias bibliográficas.

- Bioensayos Corresponde a disertaciones, sobre aspectos relacionados con la Educación en Biología.

- Biocrónicas Se refiere a la narración de una experiencia educativa relacionada con la educación en Biología, por ejemplo: salidas pedagógicas, experiencias de aula, proyectos escolares, historias de vida, entre otras. El texto debe ser preciso y claro para orientar al lector, así mismo debe tener coherencia con los contenidos desarrollados en el cuerpo del trabajo

- Biotesis: Corresponde resúmenes analíticos educativos -RAEs- de trabajos de grado de de programas de Pregrado y Especialización, así como de tesis de Maestría y Doctorado.

- Biogalerías Corresponde a una secuencia de fotografías que ilustran determinados aspectos sobre la Biología y su enseñanza.

\section{ESTRUCTURA DE LOS ESCRITOS}

\section{Para bioartículos.}

Extensión: entre 15 y 20 páginas.

Título: deberá llevar el menor número de palabras, que dé cuenta adecuadamente del contenido del escrito tanto en inglés como en español.

Resumen: Este no debe exceder las 250 palabras, donde se dé cuenta de: objetivos principales, metodología, y las conclusiones más importantes.

Abstract: Corresponde a la traducción del resumen.

Palabras clave: estas deben corresponder a conceptos necesarios para entender el cuerpo del artículo, con un máximo de cinco palabras.

Key Word: Corresponde a la traducción de las palabras clave.

${ }^{1}$ Cabe aclarar que todos los escritos que se publican en la revista hacen referencia a la Enseñanza en Biología, lo cual no obsta la publicación de resultados de investigaciones biológicas, siempre y cuando se explicite las implicaciones educativas de las mismas. 
Introducción: debe contener la naturaleza y visión general del problema, hacer evidentes la pertinencia y originalidad del estudio. De igual manera, es conveniente mencionar los objetivos y principales aportes de la investigación.

Materiales y métodos: ha de explicitar de forma breve el lugar donde se realizó la investigación, el enfoque metodológico, los materiales, instrumentos empleados para la obtención de los datos y los métodos utilizados para el análisis de los mismos.

Resultados y discusión: los resultados deben presentarse de forma clara y concisa, utilizando si es necesario figuras y tablas. Es importante destacar las implicaciones educativas.

Conclusiones: deben ser claras y concisas y originarse a partir de los resultados obtenidos, incluyendo las implicaciones educativas.

\section{Para Bioensayos}

Extensión: entre 5 y 10 páginas.

Es un escrito de disertación sobre un tema que consta de los siguientes elementos:

Título Este debe corresponder al tema de la reflexión, debe ser preciso y claro para orientar al lector, así mismo debe tener coherencia con los contenidos desarrollados en el cuerpo del trabajo, este se debe presentar tanto en inglés como en español.

Resumen: Este no debe exceder las 250 palabras, donde se dé cuenta de: objetivos principales, metodología, y las conclusiones más importantes.

Abstract: Corresponde a la traducción del resumen.

Palabras clave: estas deben corresponder a conceptos necesarios para entender el cuerpo del ensayo, con un máximo de cinco palabras.

Key Word: Corresponde a la traducción de las palabras clave.

Son elementos constituyentes de un ensayo: introducción, tesis, desarrollo y conclusiones.

- La introducción corresponde a los aspectos contemplados en el resumen.

- La tesis es la idea principal o el punto de vista que el autor argumenta a lo largo del escrito.

- El desarrollo o contenido central presenta los distintos argumentos que sustentan la idea central del escrito, estos pueden ser soportados en: hechos históricos, experiencias, principios filosóficos y planteamientos de otros autores que trabajan sobre la tesis central.

- Las conclusiones han de derivar de la discusión desarrollada a lo largo del texto.

Cabe aclarar que no es requisito presentar los elementos mencionados en forma de apartados. El autor tiene autonomía para la presentación de este escrito.

\section{Para Biocrónicas}

Extensión: entre 5 y 10 páginas.

Es un escrito que describe una experiencia educativa. Consta de los siguientes elementos:

Título Este debe corresponder a la narración de una experiencia, debe ser preciso y claro para orientar al lector, así mismo debe tener coherencia con los contenidos desarrollados en el cuerpo del trabajo, este se debe presentar tanto en inglés como en español.

Cuerpo del escrito: el estilo así como la estructura del escrito dependerá del criterio del autor. 


\section{Para Biotesis}

Corresponde al resumen de trabajos de grado y tesis de programas de formación de pregrado y postgrado. Su extensión no debe superar las tres páginas. La estructura del resumen corresponde a la del Resumen Analítico Educativo (RAE) que se presenta a continuación:

Título: deberá llevar el menor número de palabras, que dé cuenta adecuadamente del contenido del escrito tanto en inglés como en español.

Autor(es): debe colocarse la totalidad de personas que realizaron el trabajo, así como su filiación y correo electrónico.

Depósito: ha de colocarse el lugar donde se puede encontrar el informe en extenso

Institución: se debe explicitar el nombre de la Institución Educativa en la cual se dirigió el trabajo.

Palabras claves/ Key Word: estas deben corresponder a conceptos necesarios para entender el cuerpo del artículo, con un máximo de cinco palabras, tanto en inglés como en español.

Descripción: debe contener la naturaleza y visión general del problema, hacer evidentes la pertinencia y originalidad del estudio. De igual manera es conveniente mencionar los objetivos y principales aportes de la investigación.

Contenido: debe presentar de forma resumida los principales elementos de los capítulos del trabajo.

Fuentes: se debe presentar el número total de fuentes; así como explicar en forma de cita bibliografía las fuentes más relevantes de la investigación

Materiales y métodos: ha de explicitar de forma breve el lugar donde se realizó la investigación, la población objeto de estudio, el enfoque metodológico, los materiales, instrumentos empleados para la obtención de los datos y los métodos utilizados para el análisis de los mismos.

Principales Resultados: los resultados deben presentarse de forma clara y concisa.

Conclusiones: deben ser claras y originarse a partir de los resultados obtenidos, incluyendo las implicaciones educativas.

Aportes Educativos de la Investigación: ha de explicitar de forma clara los alcances que tiene la investigación y su posible impacto en el área de estudio.

\section{Para Biogalería}

Ha de incluir un título y una introducción que permita ubicar al lector sobre la intención del material fotográfico el cual debe ser de alta resolución. Las fotografías deben estar acompañadas con las respectivas leyendas o comentarios las cuales deben presentarse en la parte inferior. Así mismo, se requiere que se mencione el autor de cada fotografía.

\section{PRESENTACIÓN DE LA BIBLIOGRAFÍA}

Para efectos de la presentación de la Bibliografía, a continuación se transcriben las normas referenciadas en la Revista Colombiana de Educación.

"Debe ser confeccionada en estricto orden alfabético, según el apellido de los autores. Si hay más de un texto de un mismo autor, se pone en orden cronológico, desde el más antiguo al más nuevo. Si aparece una obra de un autor y otra del mismo autor pero con otras personas, primero se pone el del autor solo y luego el otro.

Ejemplo: Primero Jones, G. (1987) y luego Jones, G. \& Coustin, L. (1985).

En el texto impreso (artículo, libro o informe) el título de la obra principal va escrito en letra cursiva, y la referencia completa tiene sangría al margen izquierdo del texto (en la quinta letra desde el margen), desde la segunda línea de la referencia, con el propósito que el apellido del autor quede destacado. Además, el texto queda impreso o escrito con justificación completa.

Ejemplo:Alexander, P. C., Moore, S. \& Alexander, E. R. (1991). What is transmitted in the intergeneration transmission of violence? Journal of Marriage and the Family, 53, 657-668. 
Libro completo: Se pone el apellido del autor, una coma, un espacio, la inicial o iniciales del nombre seguidas de un punto (espacio entre puntos), espacio, año entre paréntesis, punto, espacio, título del libro (en letra cursiva y sólo con mayúscula la primera letra; excepciones: la primera letra después de dos puntos de un título en inglés, nombres de instrumentos, congresos o seminarios y nombres propios), punto, espacio, ciudad (en caso de USA: ciudad, estado abreviado; ejemplo: Boston, MA), dos puntos, espacio, editorial y punto. En caso de dos autores se separan por \&. En caso de más de dos autores, se separan los nombres con coma y entre el penúltimo y último se pone \&. Deben ser nombrados todos los autores, cuando son menos de 7 autores.

Ejemplo:

Jiménez, G. F. (1990). Introducción al Psicodiagnóstico de Rorschach y láminas proyectivas. Salamanca: Amarú Ediciones.

Cuando los autores son 7 ó más, se escriben los primeros 6 y luego se escribe et al. Ejemplo:

Alvarado, R., Lavanderos, R., Neves, H., Wood, P., Guerrero, A., Vera, A. et al. (1993). Un modelo de intervención psicosocial con madres adolescentes. En R. M. Olave \& L. Zambrano (Comp.), Psicología comunitaria y salud mental en Chile (pp. 213-221). Santiago: Editorial Universidad Diego Portales.

La APA exige que los títulos de obras (no la revista de publicación de esa obra) que no estén en el idioma de la revista a la cual se envía el artículo sean traducidos, entre paréntesis cuadrados, al idioma de la revista. Del ejemplo anterior: Davydov, V. V. (1972). De introductie van het begrip grootheid in de eerste klas van de basisschool [La introducción del concepto de cantidad en el primer grado de la escuela básica]. Groningen, Holanda: Wolters-Noordhoff.

Libro completo con edición diferente de la primera: se pone la edición entre paréntesis después del título, dejando sólo un espacio y en letra normal. Se abrevia ed. Ejemplo:

Nichols, M. \& Schwatz, R. (1991). Family therapy: Concepts and methods (2 $2^{\mathrm{a}}$ ed.). Boston, MA: Allyn and Bacon.

Libro completo con reimpresión: se pone el año cuando el autor escribió el libro/el año de reimpresión. Se pone la reimpresión entre paréntesis después del título, dejando sólo un espacio y en letra normal. No se abrevia.

Rorschach, H. (1921/1970). Psicodiagnóstico (7ª Reimpresión). Buenos Aires: Paidós.

Capítulo de libro: el título del capítulo va en letra normal y en primer lugar. Después del punto se pone En, espacio, inicial del nombre de los autores, editores, compiladores, espacio, apellido, coma, entre paréntesis si son editores o compiladores (se abrevia Ed. si es un editor, Eds. si es más de uno, Comp. si es o son compiladores, Trad. si son traductores), espacio, coma, espacio, título del libro (en letra cursiva), espacio, páginas del libro en las que aparece el capítulo entre paréntesis (se abrevia pp. para páginas y p. para una página, separadas por guión cuando es más de una página). Si la editorial es igual a los Eds., Compiladores, o autor se pone al final: ciudad: Autor (es), Compiladores, Editor(es).

Ejemplo:

Garrison, C., Schoenbach, V. \& Kaplan, B. (1985). Depressive symptoms in early adolescence. En A. Dean (Ed.), Depression in multidisciplinary perspective (pp. 60-82). New York, NY: Brunner/Mazel.

Artículo en Revista: El título del artículo va en letra normal y en primer lugar, espacio, nombre de la revista en letra cursiva, coma en letra cursiva, número de la revista en letra cursiva y números arábigos, coma en letra cursiva, páginas separadas por guión en letra normal y punto. La primera letra de las palabras principales (excepto artículos, preposiciones, conjunciones) del título de la revista es mayúscula.

Sprey, J. (1988). Current theorizing on the family: An appraisal. Journal of Marriage and the Family, 50, 875-890.

Cuando el número de la revista consta de más de un volumen y sólo cuando todos los volúmenes comienzan con la página 1, se pone: número de la revista en letra cursiva y números arábigos, paréntesis, volumen de la revista en letra normal, paréntesis, coma en letra normal, páginas separadas por guión en letra normal y punto. 
Castro, R. (1994). Estrategias en salud reproductiva del adolescente en Chile. Revista de la Sociedad Chilena de Obstetricia y Ginecología Infantil y de la Adolescencia, 1(2), 38-45.

Cuando la revista no tiene número, sino que sólo se expresa un mes, una estación del año o es una publicación especial, en vez del número se pone el mes en cursiva, coma en cursiva, páginas en letra normal, o publicación especial.

Ejemplo: Thompson, L. \& Walker, A. (1982). The dyad as the unit of analysis: Conceptual and methodological issues. Journal of Marriage and the Family, November, 889-900.

Artículo en prensa o enviado para su publicación: si un artículo está en prensa, es porque ya ha sido aceptado por la revista para su publicación, que puede ser en fecha muy próxima. En este caso, en vez del año se pone (en prensa), y no se pone ni el volumen ni páginas de la revista, pero sí el título de la misma.

Ejemplo: Bourgeois, E. (en prensa). Evaluer la transformation de structures de connaissances propositionnelles chez les adultes en formation. Questions méthodologiques pour la recherche. Psychologie.

Si el artículo ha sido enviado a una revista, pero aún está en revisión y todavía no ha sido aceptado para ser publicado, se pone al final Manuscrito enviado para publicación y no se pone el nombre de la revista. El año corresponde al del artículo.

Resumen (Abstract) de Artículos: Cuando la referencia es un resumen o abstract de la fuente original, debe ponerse la palabra Resumen o Abstract entre paréntesis cuadrados después del título:

Chalon, S., Delion-Vancassel, S., Belzung, C., Guilloteau, D., Leguisquet, A. M., Besnard, J. C. et al. (1998). Dietary fish oil affects monoaminergic neurotransmission and behavior in rats [Abstract]. The Journal of Nutrition, 128, 2512-2519.

Cuando la referencia es un resumen o abstract de una fuente secundaria, debe citarse la fuente secundaria y la fecha de extracción: Slate, J. R. (1998). Sex differences in WISC-III IQs: Time for separate norms? Journal of Psychology, 132, 677-679. Abstract extraido el 31 de enero, 2000 de la base de datos de PsycINFO: 1996-1998, 2000, Abstract 1998-11886-010.

Artículo en el periódico o revista de circulación masiva: Se pone el día y mes después del año, separados por una coma, punto, el título del artículo en letra normal, punto, el nombre del periódico o revista en letra cursiva, coma en letra cursiva, espacio, p. y el número de la página. Si son más de una página y son seguidas, se pone pp. A1-A2. Si no son seguidas, se separan por coma.

Ej.: pp. A1, A4. Artaza, J. (1995, Abril 13). Juventud y vocación. El Mercurio, p. C1. Si el artículo no tiene autor, el título reeemplaza al autor.

Ej: El temor en los niños. (2002, Enero 19). Las Ultimas Noticias, p. 14. Para efectos del orden alfabético, se considera "temor" y no "El". Si las páginas fueran discontinuadas, se separan con una coma. Ej.: pp. 14, 25. Si se trata de una carta al editor de un periódico, se pone después del título Carta al editor entre paréntesis cuadrados. Argentina desde adentro [Carta al editor]. (2002, Enero 19). Las Ultimas Noticias, p. 14.

Informes o Estudios seriados: Se pone la serie y número entre paréntesis después del estudio específico o después de Estudios si es una serie. Cuando el editor es el mismo que el autor, después de los dos puntos que siguen a la ciudad se pone autor o autores, según lo que corresponda.

Ej: Centro Latinoamericano de Demografía (1991). Recursos humanos en salud: Bolivia y Ecuador (LC/DEM/R. 165, Serie A. No 259). Santiago: Autor.

Libro o informe de alguna institución: La institución no se abrevia ni se usan siglas. Después del nombre de la institución va un punto. Si es un Ministerio, como todos tienen nombres parecidos, primero se pone el país, coma, espacio, nombre del ministerio. 
Ej: Organización Mundial de la Salud. (1989). Trastornos mentales y del comportamiento. En Organización Mundial de la Salud (Ed.), Décima revisión internacional de la clasificación internacional de enfermedades (Versión española del Borrador para Estudios de Campo $\mathrm{N}^{\mathbf{0}}$ 4). Madrid: Editor.

Diccionarios o enciclopedias: el nombre del autor o editor (Ed. entre paréntesis cuando es editor). Después del título del diccionario o enciclopedia y después de un espacio se pone entre paréntesis la edición si no es la primera, coma y el volumen consultado. Cuando es un diccionario o enciclopedia con un equipo editorial extenso, se pone sólo al editor principal, seguido por et al.

Ej:Sadie, S. (Ed.). (1980). The new Grove dictionary of music and musicians (6 ${ }^{\mathrm{a}}$ ed., Vol 15). Londres:Macmillan.

Informes técnicos o de investigaciones de universidades o centros de investigación: después del título del informe se pone el nombre del proyecto entre paréntesis (si existe). Al final la ciudad, coma, espacio, país, dos puntos, espacio, Universidad, coma, espacio, Departamento, Facultad o Escuela, espacio, y punto. Ej:Milicic, N., Alcalay, L. \& Torretti, A. (1992). Diseño de un programa para favorecer la identidad femenina en alumnas de $7^{\circ}$ y $8^{\circ}$ año de educación general básica (Proyecto FONDECYT 1992/0799). Santiago: Pontificia Universidad Católica de Chile, Escuela de Psicología.

Ponencias o conferencias en simposio, congreso, reuniones, etc: si la contribución está publicada en un libro con editor se debe señalar la publicación. El título del simposio, congreso o reunión debe ir con mayúsculas.

Ej:Deci, E. L. \& Ryan, R. M. (1991). A motivvational approach to self: Integration in personality. En R. Dienstbier (Ed.), Nebraska Symposium on Motivation: Vol. 38. Perspectives on motivation (pp. 237-288). Lincoln: University of Nebraska Press.

Si la contribución no está publicada, se pone el mes en el que tuvo lugar el evento, separado de una coma después del año. Después del título de la ponencia o conferencia se pone Ponencia presentada en, el nombre completo del congreso con las palabras principales en mayúscula, coma, espacio, ciudad, coma, espacio, país, punto. Si fue poster se pone Poster presentado en....

Si es un trabajo presentado en un Simposio o en una Mesa Redonda, debe señalarse el título del mismo y el nombre del Coordinador.

Ej: Cumsille, P. \& Graham, J. (2001, julio). Modelaje de curvas de crecimiento en la evaluación de programas de prevención de consumo de alcohol y drogas. En P. Cumsille (Coordinador), Methodological strategies for the study of psychological processes: Applications to the study of alcohol and drug use. Simposio realizado en XXVIII Congreso Interamericano de Psicología, Santiago, Chile.

Trabajo de grado o tesis de maestría o doctorado: tesis para optar al título de..., Escuela o Departamento, Universidad, ciudad, país.

Alamos, F. (1992). Maltrato infantil en la familia: tratamiento y prevención. Memoria para optar al Título de Psicólogo, Escuela de Psicología, Pontificia Universidad Católica de Chile, Santiago, Chile.

Cuando se trata de una disertación doctoral que está publicada, debe señalarse la universidad y año de la disertación, así como el volumen y páginas de la obra donde está publicada.

Ross, D. F. (1990). Unconscious transference and mistaken identity: When a witness misidentifies a familiar but innocent person form a lineup (Disertación doctoral, Cornel University, 1990). Dissertation Abstracts International, 51, 417.

Cuando la disertación doctoral no está publicada, debe señalarse que no está publicada, la universidad, ciudad y país y año de la disertación.

Ejemplo: Wilfley, D. E. (1989). Interpersonal analyses of bulimia: Normal weight and obese. Disertación doctoral no publicada, University of Missouri, Columbia, Estados Unidos. Si la tesis de magister no está publicada, debe señalarse: Cumsille, P. (1992). Family adaptability, family cohesion, social support, and adolescent depression: Analysis of a sample of families attending an outpatient clinic. Tesis de Magister no publicada, University of Maryland, College Park, Maryland, Washington, DC, Estados Unidos.

Manuscrito no publicado: después del título se pone Manuscrito no publicado. Ejemplo: Bringiotti, M. I. \& Barbich, A. (1992). Adaptación y validación del Child Abuse Potential Inventory - CAP. Versión 
preliminar para la Argentina. Manuscrito no publicado. Si los autores pertenecen a alguna institución o universidad, se señala ésta, la ciudad y el país.

Medios audiovisuales: estos pueden ser películas, programas de TV, video o cualquier otro medio audiovisual. En general, se debe señalar al productor o director, o ambos, poner en paréntesis cuadrados el tipo de medio y la ciudad de origen (en el caso de las películas, se pone el país de origen) Ejemplos: Scorsese, M. (Productor) \& Lonergan, K. (Escritor/Director). (2000)). You can count on me [Película]. Estados Unidos: Paramount Pictures.

Medios electrónicos en Internet: si es un artículo que es un duplicado de una versión impresa en una revista, se utiliza el mismo formato para artículo de revista, poniendo entre paréntesis cuadrados [Versión electrónica] después del título del artículo: Maller, S. J. (2001). Differential item functioning in the WISCIII: Item parameters for boys and girls in the national standardization sample [Versión electrónica]. Educational and Psychological Measurement, 61, 793-817.

Si el artículo en línea pareciera ser algo distinto de la versión impresa en una revista, después de las páginas de la revista, se pone la fecha de la extracción y la dirección: Hudson, J. L. \& Rapee, M. R. (2001). Parent ${ }^{-}$child interactions and anxiety disorders: An observational study. Behaviour Research and Theraphy, 39, 1411-1427. Extraido el 23 Enero, 2002, de http://www.sibuc.puc.cl/sibuc/index.html

Si el artículo aparece sólo en una revista de Internet: Biglan, A. \& Smolkowski, K. (2002, Enero 15). The role of the community psychologist in the $21^{\text {st }}$ century. Prevention \& Treatment, 5, Artículo2. Extraido el 31 Enero, 2002 de http://journals.apa.org/prevention/volume5/pre0050002a.html

Cuando se trata de un capítulo o sección de un documento de Internet de un sitio Web de una universidad: se debe identificar la organización y luego la dirección exacta donde se encuentra el documento. En vez de páginas del capítulo leído, se anota el número del capítulo. Jencks, C. \& Phillips, M. (1999). Aptitude or achievement: Why do test scores predict educational attainments and earnings? En S. E. Mayer \& P. E. Peterson (Eds.) Earning and learning: How schools matter (cap. 2). Extraido el 31 Enero, 2002 del sitio Web de Columbia University: http://www.columbia.edu/cu/lweb/indiv/ets/offsite.html\#finding y luego http://brookings.nap.edu/books/0815755295/html/15.html\#pagetop"

\section{CITACIÓN DE BIBLIOGRAFÍA EN EL TEXTO.}

A continuación se presentan algunos ejemplos para citar con normas APA dentro del texto:

... Bustillo (1998) si el nombre del autor es parte de la oración.

... Tróchez y Rodríguez (1989) si los nombres de los dos autores son parte de la oración.

López et al. (1989) si son 3 o más autores y sus nombres hacen parte de la oración.

... (Gutiérrez 1999) si la cita va al final de la frase.

... (Bustillo y Rodríguez 1999) si la cita va al final de la frase.

... (Ramírez et al. 1999) si se cita una publicación con 3 o más autores al final de la frase.

... (Bueno 1998, 1999) para dos artículos del mismo autor.

... (Portilla 1998 a, 1998 b) para dos artículos del mismo autor en el mismo año

... (Gutiérrez 1987; Rodríguez 1998; Ramírez 1999) citación múltiple, separados por punto y coma y en orden ascendente de año.

... (Parra, en prensa). En la literatura citada es necesario señalar el nombre de la Revista donde va a publicarse el artículo.

... (P. Reyes, com. pers.). Es necesario que el autor obtenga permiso para esta citación. Puede señalarse bien sea como pie de página o en el listado de Literatura citada, indicando la fecha de la comunicación. 